CNS \& NEUROLOGICAL DISORDERS-DRUG TARGETS 14:(3) pp. 350-359. (2015)

\title{
Effect of probenecid on the pain-related behaviour and morphological markers in orofacial formalin test of the rat
}

\section{Running title: Probenecid in orofacial formalin test}

Annamária Fejes-Szabó M.Sc. ${ }^{1}$, Zsuzsanna Bohár M.Sc. ${ }^{1,2}$, Gábor Nagy-Grócz M.Sc. ${ }^{1}$, Enikő Vámos M.Sc., Ph.D. ${ }^{1}$, Lilla Tar M.D. ${ }^{1}$, Borbála Pödör M.Sc. ${ }^{3}$, János Tajti M.D., Ph.D. ${ }^{1}$, József Toldi M.Sc., Ph.D., D.Sc. ${ }^{2,4}$, László Vécsei M.D., Ph.D., D.Sc. ${ }^{1,2, \# *}$ and Árpád Párdutz M.D., Ph.D. ${ }^{1, \#}$

${ }^{1}$ Department of Neurology, Faculty of Medicine, Albert Szent-Györgyi Clinical Centre, University of Szeged, Semmelweis u. 6., Szeged, H-6725, Hungary

${ }^{2}$ MTA-SZTE Neuroscience Research Group, Semmelweis u. 6., Szeged, H-6725, Hungary

${ }^{3}$ Department of Physiology \& Biophysics, Faculty of Medicine, Dalhousie University, Halifax, Nova Scotia, B3H 1X5, Canada

${ }^{4}$ Department of Physiology, Anatomy and Neuroscience, University of Szeged, Közép fasor 52., Szeged, H-6726, Hungary

\# These authors contributed equally to this work.

* Corresponding author:

László Vécsei M.D., Ph.D., D.Sc.

Department of Neurology, Faculty of Medicine, Albert Szent-Györgyi Clinical Centre, University of Szeged, Hungary

Semmelweis u. 6, H-6725 Szeged, Hungary

Tel.: +36 62545351 
Fax: +36 62545597

E-mail: vecsei.laszlo@med.u-szeged.hu 


\section{Abstract}

Probenecid has been widely used in the treatment of gout, but evidence suggest that it may also have anti-nociceptive effects in different inflammatory and pain conditions. We examined the potential modulatory effects of probenecid on behavioural and morphological markers in the orofacial formalin test of the rat.

One hour after pre-treatment with vehicle or probenecid ( $1 \mathrm{mmol} / \mathrm{kg}$ body weight) intraperitoneally, $50 \mu 1 \quad 1.5 \%$ formalin solution or physiological saline was injected subcutaneously into the right whisker pad of rats. The rubbing activity directed to the injected whisker pad was then measured for a period of 45 minutes. Four hours after formalin injection, the caudal part of spinal trigeminal nucleus was removed and subjected to c-Fos and neuronal nitric oxide synthase (nNOS) immunohistochemistry and to interleukin-1ß and NAD(P)H:quinone oxidoreductase 1 (NQO1) Western blot.

There was a significant decrease in formalin-induced biphasic behavioural response and c-Fos and nNOS immunoreactivity in the rats who were pre-treated with probenecid. However there were no alterations in expression of interleukin-1 $\beta$ or NQO1 after formalin administration.

Our results suggest that probenecid has an anti-nociceptive effect in the trigeminal inflammatory pain model. This effect may be through influencing the release of prostaglandin E2 or desensitizing the transient receptor potential channel subtype A member 1 or the transient receptor potential channel subtype $V$ member 2 or the effect may be through modulating kynurenic acid levels in the central nervous system. Thus, probenecid might be a potential candidate for the treatment of trigeminal activation related pain conditions. 
Key words: caudal part of spinal trigeminal nucleus; c-Fos; interleukin-1及; NAD(P)H:quinone oxidoreductase 1; neuronal nitric oxide synthase; orofacial formalin test; probenecid- 


\section{Introduction}

Probenecid (PROB, $p$-(di- $n$-propylsulfamyl)benzoic acid) has primarily been used to enhance the renal retention of penicillin and to treat gout, due to its ability to increase the renal excretion of uric acid . Besides its effects on the kidney, research data suggest that PROB may have anti-inflammatory and anti-nociceptive properties as well. PROB blocks the multidrug resistance-associated protein 4 (MRP4), an organic anion transporter, which is crucial in the release of key inflammatory mediators such as prostaglandin E1 and prostaglandin E2 (PGE2). In addition, PROB inhibits the pannexin-1 channel, which may be relevant in neuronal inflammatory processes . On the other hand, PROB is an agonist of the transient receptor potential channel subtype A member 1 (TRPA1) and the transient receptor potential channel subtype $\mathrm{V}$ member 2 (TRPV2), which play an important role in pain sensation . It is also well known, that an agonist-mediated desensitization of these TRPA1 and TRPV2 can be induced, leading to analgesia. These results strongly suggest that PROB might be effective in mitigating inflammation and nociception. In contrast, Bang and co-workers raise the possibility that PROB can elicit the pain induced by inflammation through its agonism on TRPV2 .

Fundamental research and pharmacological studies investigating the trigeminal inflammation and nociception often use the orofacial formalin test, a reliable model for studying somatic pain causing the activation and sensitization of trigeminal system , which is crucial in the pathomechanism of headaches such as migraine - one of the most common neurological disorders . Formalin solution administered subcutaneously into the upper lip gives rise to a tissue injury, nociception and inflammation, activating the primary nociceptors, enhancing the immunoreactivity of c-Fos, a marker of neuronal activation in the caudal part of spinal trigeminal nucleus (TNC) and resulting in an increase in neuronal 
nitric oxide synthase (nNOS), which is a suggested marker of central sensitization . The induction of formalin causes a biphasic nociceptive behavioural response consisting of rubbing acitivity during an early and short-lasting (3-4 min) first phase of rubbing is followed by a tonic prolonged (20-22 $\mathrm{min}$ ) second phase, the phases being separated by a 910 min quiescent period. The first phase is thought to be due to the direct chemical stimulation of the nociceptors by the formalin solution, while the second phase is a manifestation of the processes induced in the spinal cord during the first phase, together with peripheral inflammation. The inflammatory mediators such as PGE2 and interleukin-1 (IL-1 $\beta$ ) and the oxidative stress may have important role in the formation of changes induced by subcutaneous (s.c.) formalin injection .

Based on previous experimental results, it has been proposed that PROB, through its different receptorial actions, may have anti-nociceptive effect that can be seen in the orofacial formalin test. Thus the aim of the current study was to investigate whether PROB exerts a modulatory effect in this tonic trigeminal pain model through the examination of behavioural and morphological markers. 


\section{Materials and Methods}

\section{Animals:}

The procedures used in this study followed the guidelines of the eighth edition of the Guide for the Care and Use of Laboratory Animals and the Use of Animals in Research of the International Association for the Study of Pain and the directive of the European Economic Community (86/609/ECC). They were approved by the Committee of Animal Research at the University of Szeged (I-74-12/2012) and the Scientific Ethics Committee for Animal Research of the Protection of Animals Advisory Board (XXIV./352/2012.). Sixty adult male Sprague-Dawley rats weighing 200-250 g were used. The animals were raised and housed under standard laboratory conditions (in an air-conditioned, humidity-controlled and ventilated room), with drinking water and regular rat chow available ad libitum on a $\mathbf{1 2 h - 1 2 h}$ dark-light cycle. Each rat was used only once.

\section{Drug administration:}

The rats were divided into two groups ( $\mathrm{n}=\mathbf{3 0}$ per group). The animals in the Placebo group received only intraperitoneal vehicle solution (physiological saline, $1.5 \mathrm{ml}$ ) as pre-treatment. In the PROB group, the rats were pretreated with an intraperitoneal injection of PROB (Sigma-Aldrich; $1 \mathrm{mmol} / \mathrm{kg}$ body weight, diluted to $1.5 \mathrm{ml}, \mathrm{pH} 7.4$ ). Both groups of animals were divided further into two subgroups $(n=15)$ : 1 hour after the PROB or vehicle pretreatment, half of the animals received a s.c. injection of $50 \mu 11.5 \%$ formalin solution $(0.55 \%$ formaldehyde, Sigma-Aldrich) diluted in physiological saline, administered via a 26-gauge needle into the right whisker pad (Placebo-Form and PROB-Form), while the other half of 
the rats were injected with s.c. $50 \mu 1$ physiological saline without formalin (Placebo-Phys and PROB-Phys).

\section{Behavioural test:}

The testing procedures were performed during the light phase (between 8 a.m. and 2 p.m.) in a quiet room. The test box was a $30 \times 30 \times 30 \mathrm{~cm}$ glass terrarium with mirrored walls. For the off-line analysis of rubbing activity directed to the whisker pad, the behaviour of the individually tested rats was recorded with a video camera (Logitech HD Webcam C615) situated $1 \mathrm{~m}$ above the terrarium. One hour after pre-treatment with vehicle $(\mathbf{n}=20)$ or PROB (n=20) and after a 10-min habituation in the test box, the whisker pads of the rats were injected with s.c. formalin ( $\mathbf{n}=\mathbf{1 0}$ per subgroup) or physiological saline $(\mathbf{n}=\mathbf{1 0}$ per subgroup) and the animals were replaced immediately back in the chamber for $45 \mathrm{~min}$. The rats did not receive any food or water during the observation period. The test box was cleaned and decontaminated after each animal. An observer blind to the experimental procedures analysed the recorded videos. The 45-min recording period was divided into $15 \times 3$-min blocks and the total time (number of seconds) spent on rubbing the injected area with the ipsilateral fore- or hindpaw was measured in each block and defined as the nociceptive score for that block. Earlier literature findings led us to use the grooming activity as control, measured in animals that received s.c. physiological saline .

\section{4. c-Fos and nNOS immunohistochemistry:}

Four hours after the formalin or physiological saline injection, the rats $(\mathbf{n}=\mathbf{5 - 9}$ per subgroup) were perfused transcardially with $100 \mathrm{ml}$ phosphate-buffered saline (PBS), followed by $500 \mathrm{ml} \mathrm{4 \%}$ paraformaldehyde (Merck) in phosphate buffer under deep chloral 
hydrate (0.4 g/kg body weight, Sigma-Aldrich) anaesthesia. The medullary segment containing the TNC between +1 and $-5 \mathrm{~mm}$ from the obex was removed, postfixed overnight for immunohistochemistry in the same fixative and cryoprotected (10\% sucrose for $2 \mathrm{~h}, 20 \%$ sucrose until the blocks sank, and 30\% sucrose overnight). Before sectioning, each segment was marked with a small incision on the ventral and left (contralateral) side of the tissue block, allowing side discrimination during the quantification process. $30-\mu \mathrm{m}$ transverse cryostat sections were cut through the rostrocaudal axis from the beginning of the TNC and were serially collected in wells containing cold PBS. Each well contained every tenth section at $0.3-\mathrm{mm}$ intervals along the rostrocaudal axis (15 levels/sections). The free-floating sections were rinsed in PBS and immersed in $0.3 \% \mathrm{H}_{2} \mathrm{O}_{2}$ (Scharlau Chemie) in PBS for 30 min to suppress endogenous peroxidase activity. After several rinses in PBS containing 1\% Triton X-100 (PBST; VWR International), sections were incubated at room temperature overnight in PBST containing rabbit anti-rat c-Fos polyclonal antibody (Santa Cruz Biotechnology, sc-52) at a dilution of 1:2000 or at $4^{\circ} \mathrm{C}$ for two nights in PBST containing rabbit anti-rat nNOS polyclonal antibody (EuroProxima, B220-1) at a dilution of 1:5,000. The immunohistochemical reaction was visualized by using Vectastain Elite avidinbiotin kits (Vector Laboratories, PK6101). Briefly, the sections were incubated at room temperature for 2 hours in PBST containing goat anti-rabbit biotinylated secondary antibody. After several rinses in PBST, and incubation at room temperature for 2 hours in PBST containing avidin and biotinylated horseradish peroxidase, the sections were stained with 3,3'-diaminobenzidine (Sigma-Aldrich) intensified with nickel ammonium sulphate (Scharlau Chemie). The specificity of the immune reactions was checked by omitting the primary antiserum.

The counting of immunoreactive (IR) cells in the TNC was performed by an observer blind to the experimental procedures under the 10x objective of a Nikon Optiphot-2 light 
microscope in every tenth transverse section in each animal. Before the counting, the location of each section along the rostrocaudal axis and the location of the TNC on each medullary section were determined by means of The Rat Brain in Stereotaxic Coordinates Atlas. The c-Fos neurones with obvious specific nuclear staining and the nNOS-IR neurones with cytoplasmic and dendritic staining and a nucleus were taken into consideration and were counted in the TNC both ipsilaterally and contralaterally to the formalin or physiological saline injection.

\section{IL-1ß and NAD(P)H:quinone oxidoreductase 1 (NQO1) Western blot:}

Four hours after the formalin or physiological saline injection, the rats $(n=5$ per subgroup) were deeply anaesthetized with chloral hydrate as described above, perfused transcardially with $100 \mathrm{ml}$ ice cold PBS and the ipsi- and contralateral side of the TNC between 0 and $-4 \mathrm{~mm}$ from the obex was removed. Until measurements, the samples were stored at $-80^{\circ} \mathrm{C}$. The TNC segments were sonicated in ice cold lysis buffer containing $50 \mathrm{mM}$ Tris-HCl, $150 \mathrm{mM} \mathrm{NaCl}, 0.1 \%$ igepal, $0.1 \%$ cholic acid, $2 \mu \mathrm{g} / \mathrm{ml}$ leupeptin, $2 \mathrm{mM}$ phenylmethylsulphonyl fluoride (PMSF), $1 \mu \mathrm{g} / \mathrm{ml}$ pepstatin, $2 \mathrm{mM}$ EDTA and $0.1 \%$ sodium dodecyl sulphate (SDS) (all chemicals were from SigmaAldrich). The lysates were cleared from cellular debris by centrifugation at 12,000 RPM for $10 \mathrm{~min}$ at $4^{\circ} \mathrm{C}$ and supernatants were aliquoted and stored at $-20^{\circ} \mathrm{C}$. Protein concentration was measured according to BCA protein assay method with BCA Protein Assay Kit (Novagen) using bovine serum albumin as a standard. Samples were cooled on ice during the whole procedure. Prior to loading into the gels, each sample was mixed with sample buffer, and denaturated by boiling for 3 min. Equal amounts of protein samples $(20 \mu g /$ lane $)$ were separated by standard SDS polyacrylamide gel electrophoresis (SDS-PAGE) on 12\% Tris-Glycine gel and electrotransferred onto 
Amersham Hybond-ECL nitrocellulose membrane (0.45 $\mu \mathrm{m}$ pore size, GE Healthcare). The Page Ruler Prestained Protein Ladder (Fermentas, 10-170 kDa) was used to determine approximate molecular weights. Following the transfer, membranes were blocked for one hour at room temperature in Tris-buffered saline containing Tween 20 (TBST, MP Biomedicals) and 5\% non fat dry milk powder and then incubated in TBST containing $1 \%$ non fat dry milk and (i) rabbit anti-rat IL-1ß (H-153) polyclonal antibody (Santa Cruz Biotechnology, sc-7884, dilution: 1:200, incubation: overnight at $4^{\circ} \mathrm{C}$ ) or (ii) rabbit anti-rat NQO1 (H-90) polyclonal antibody (Santa Cruz Biotechnology, sc-25591, dilution: 1:200, incubation: overnight at $4^{\circ} \mathrm{C}$ ) or (iii) mouse anti-rat $\beta$-actin monoclonal antibody (Calbiochem, CP01, dilution: 1:100,000, incubation: overnight at $4^{\circ} \mathrm{C}$ ). Next day after several rinses in TBST, membranes were incubated in TBST containing $1 \%$ non fat dry milk and horseradish peroxidaseconjugated anti-rabbit or anti-mouse secondary antibody (Santa Cruz Biotechnology; sc-2030 and sc-2031) for 2 hours at room temperature. Protein bands were visualized after incubation of membranes with the SuperSignal West Pico Chemiluminescent Substrate (Pierce) using Carestream Kodak BioMax Light film (Kodak).

For densitometric analyses, films were scanned and quantified using Java ImageJ $1.47 \mathrm{v}$ analysis software (National Institutes of Health). The $\beta$-actin was used as sample loading control and normalization protein as well. The results from each animal were further normalized to its own contralateral side.

\section{Statistical analysis:}

In the behavioural study, comparisons were made of the rubbing activity in each block of 3min in each of the subgroups (Placebo-Form, Placebo-Phys, PROB-Form and PROB-Phys) by using two-way repeated measures ANOVA. Subgroups were used as the between-subject 
factor and 15 blocks of 3-min as the within-subject factor for the analysis. When Mauchly's test of sphericity proved significant, the Greenhouse-Geisser correction was performed. Pairwise comparisons of subgroup means were based on the estimated marginal means with Sidak adjustment for multiple comparisons.

To compare statistical differences between subgroups in the first and second phases, the two phases were determined on the basis of significant results detected during two-way repeated measures ANOVA described above. The first phase was found to be the first of the 15 blocks, while the second phase was the sum of the fifth to the eleventh blocks. To determine significant differences between subgroups in the first or second phase, we used one-way ANOVA followed by the Tamhane post hoc test.

In terms of the immunoreactivity, the numbers of IR neurones in the various subgroups (Placebo-Form, Placebo-Phys, PROB-Form and PROB-Phys) were compared at each level of 0.3-mm (15 levels) along the rostrocaudal axis by using two-way repeated measures ANOVA. Since there was no significant difference in the number of c-Fos- and nNOS-IR neurones between the contralateral sides in the subgroups injected with s.c. formalin and the contra- and ipsilateral sides in the subgroups injected with s.c. physiological saline (data not shown), in the course of the statistical analysis the data obtained from the contralateral sides of the subgroups injected with s.c. formalin were used as controls. The contra- and ipsilateral sides of the formalin-injected subgroups were used as the between-subject factor, and the 15 levels along the rostrocaudal axis as the within-subject factor for the analysis. When Mauchly's test of sphericity proved significant, the Greenhouse-Geisser correction was performed. Pairwise comparisons of subgroup means were based on the estimated marginal means with Sidak adjustment for multiple comparisons.

Statistical analysis of the Western blot data was carried out using one-way ANOVA followed by the Sidak post hoc test. Since there was no significant difference in band 
densities between the contralateral sides in the subgroups injected with s.c. formalin and the contra- and ipsilateral sides in the subgroups injected with s.c. physiological saline (data not shown), in the course of the statistical analysis the data obtained from the contralateral sides of the subgroups injected with formalin were used as controls.

All tests were two-sided, and probability levels $\mathrm{p}<0.05$ were considered to be statistically significant. Group values are reported as means \pm S.E.M.

Statistical analysis of measurements was carried out with IBM SPSS Statistics, version 20 (IBM Corporation) software. 


\section{Results}

\section{Nociceptive response}

The behavioural pattern observed in the rats is in accordance with previous findings . After the formalin injection, the rats immediately withdrew their heads, often accompanied by vocalization. Following their return to the observation box, the rats started to rub their whisker pad continuously and intensely with the ipsilateral forepaw accompanied often by the contralateral forepaw, and occasionally scraped the perinasal area with the ipsilateral hindpaw after a period of $\sim 20$ sec. This period, referred to as the first phase, lasted $\sim 3-4$ min, and was followed by a quiescent period of 9-10 min, separating the first phase from the second phase. The second phase was characterized by less intense, but continuous rubbing of the face, predominantly with the ipsilateral forepaw consorted often by the contralateral forepaw as well. This tonic phase lasted $\sim 20-22$ min. In the PROB-Form subgroup, the behavioural pattern in both phases was clearly less pronounced than that in the Placebo-Form subgroup, and such behaviour was not witnessed at all in the Placebo-Phys and PROB-Phys subgroups, where the animals displayed very little rubbing/grooming activity.

The comparison of the 3-min blocks revealed significant differences in rubbing activity between the Placebo-Form and Placebo-Phys subgroups in the first $(* * * p<0.001)$ and fifth to eleventh $\left(* * \mathrm{p}<0.01 ;{ }^{* * *} \mathrm{p}<0.001\right)$ blocks (Fig. 1). The PROB pre-treatment significantly decreased the formalin-induced nociceptive behaviour in each block $\quad(\# p)<0.01$; $\# \#$ p $<0.001$; Fig. 1). However, it should be noted that PROB did not attenuate the effect of formalin completely in the first block, where the time spent on rubbing was still significantly higher than that in the Placebo-Phys subgroup $\left({ }^{*} p<0.05\right.$; Fig. 1). There was no significant difference between the Placebo-Phys and PROB-Phys animals in any block, nor was between the PROB-Form and PROB-Phys subgroups in any other block apart from the first (Fig. 1). 
The face rubbing activity in the Placebo-Form subgroup was significantly higher during both the first $(* * * p<0.001)$ and the second phase $(* * * p<0.001)$ than that in the PlaceboPhys subgroup (Fig. 2). PROB significantly decreased the nociceptive behaviour in both phases $(* * p<0.01 ; * * * p<0.001$; Fig. 2), however this effect was not complete in the first phase $(* p<0.05$; Fig. 2).

\section{2. $c$-Fos in the TNC}

Microscopic examination of the immunostained transverse sections of the TNC revealed c-Fos immunoreactivity in the nuclei of the neurones. In the Placebo-Form subgroup, unilateral s.c. formalin injection produced an increase in the number of c-Fos-IR neurones in the dorsal, superficial area of the transverse sections of the ipsilateral TNC as compared with the non-treated contralateral side (Fig. 3/A). This increase was significant at different levels along the rostrocaudal axis (between -0.3 and $-3.3 \mathrm{~mm}$ ), in accordance with the somatotopic representation $(* * \mathrm{p}<0.01 ; * * * \mathrm{p}<0.001$; Fig. 3/C).

In the PROB-Form subgroup, the number of c-Fos-IR neurones at the different levels along the rostrocaudal axis also increased in the ipsilateral TNC. This effect was similar, but less pronounced than that in the Placebo-Form subgroup (Fig. 3/B), i.e. PROB significantly decreased the formalin-related activation of the second order trigeminal neurones at several levels of the TNC (between -0.3 and -2.4 mm; ${ }^{\#} p<0.05$; ${ }^{\#} \mathrm{p}<0.01$; ${ }^{\# \#} \mathrm{p}<0.001$; Fig. 3/C). On the contralateral sides of the TNCs, there were no significant differences either between the subgroups or between the different levels along the rostrocaudal axis (Fig. 3). 


\section{3. $n N O S$ in the TNC}

On the immunostained transverse sections, the nNOS-IR neurones showed cytoplasmic and dendritic staining. The unilateral s.c. formalin injection gave rise to an increase in the number of nNOS-IR neurones in the dorsal, superficial area of the ipsilateral TNC compared to the non-treated contralateral side in the Placebo-Form subgroup (Fig. 4/A). Along the rostrocaudal axis, significant increase can be observed at different levels (between -2.1 and -2.7 and between -3.3 and $-3.9 \mathrm{~mm}$; $p<0.05$; ${ }^{* *}$ p $<0.01$; Fig. 4/C). In the PROB-Form subgroup, there was no difference in the number of nNOS-IR neurones between ipsilateral and contralateral TNC (Fig. 4/B), i.e. PROB significantly decreased the number of the second order trigeminal neurones expressing nNOS at several levels of the TNC (between -2.1 and -2.7 and between -3.3 and -3.9 mm; ${ }^{\#}<0.05 ;{ }^{\# \#}<0.01$; Fig. 4/C) at the injected side. On the contralateral sides of the TNCs, there were no significant differences either between the subgroups or between the different levels along the rostrocaudal axis (Fig. 4).

\section{IL-1B and NQO1 in the TNC}

The Western blot analysis of the TNC segments did not show significant difference between the contra- and ipsilateral sides for any of the subgroups either in the case of

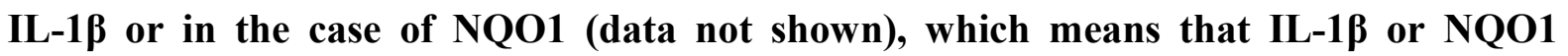
expression did not change four hours after the unilateral s.c. formalin injection and PROB pre-treatment did not have any modulatory effect on the expression of IL-1 $\beta$ or of NQO1. 


\section{Discussion}

This is the first study showing that PROB produced a pronounced anti-nociceptive behavioural effect in the orofacial formalin test especially in the second phase. PROB exhibited no effect on the baseline activity of the control animals, which received a s.c. saline injection in the whisker pad. In addition to the modulation of the nociceptive behaviour, PROB also mitigated the formalin induced c-Fos and nNOS expression in the TNC considered markers of activation and sensitization of these neurones .

This anti-nociceptive effect of PROB along with its inhibitory effect on the trigeminal activation and sensitization can be the result of various mechanisms:

PROB inhibits MRP4, an organic anion transporter, which releases key inflammatory mediators such as prostaglandin E1 and PGE2 from cells in a time- and ATP-dependent manner, and these mediators contribute to inflammation and hyperalgesia. PGE2 released from cultured rat trigeminal ganglion nerve cells can sensitize transient receptor potential channel subtype V member 1 (TRPV1) (a key factor in the development of sensitization during nociception ). Moreover, PGE2 can induce calcitonin gene-related peptide (CGRP) release (a general event after the activation of first order trigeminal neurones ) from cultured trigeminal ganglion cells . PGE2 can also act on the trigeminal system at the level of the central nervous system by enhancing the capsaicin-induced CGRP release in slice preparations of the TNC . The expression of cyclooxygenase-2 (an inducible enzyme of prostaglandin synthesis ) has also been detected in the neurones and glial cells in the trigeminal ganglion and in the neurones of the $\mathrm{TNC}$. In addition, prostaglandin receptors play a relevant role in trigeminal nociception and in the pathomechanism of trigeminal activation. They are present in the trigeminal ganglion, co-expressed with TRPV1, with a functional interaction between them in the trigeminal system, and are involved in mediating 
CGRP release from cultured rat trigeminal neurones . Quantitative autoradiography has revealed that TNC has a moderate density of PGE2 binding sites as well, and the mRNA of all prostaglandin receptors are also present there . Accordingly, a possible mechanism of action of PROB is the inhibition of the release of PGE2, a key player in trigeminal nociception and an important molecule in migraine genesis through the blockade of MRP4.

PROB also inhibits the pannexin-1 channel, which may be relevant in inflammation and hence in pain processing. The pannexin-1 channels are mainly expressed in the spinal cord ; moreover, they are present in the postsynaptic neurones and are crucial in caspase-1 activation, which leads to the production and release of interleukin-1beta (IL-1 $\beta$ ) in neurones, astrocytes and macrophages. The release of IL-1 $\beta$ from trigeminal ganglion satellite cells may contribute to the mechanisms underlying trigeminal inflammatory hyperalgesia . Upregulation of astrocytic IL-1 $\beta$ in the TNC can lead to central sensitization via its receptors present on trigeminal neurones in the same area. The interleukin-1 receptor antagonists were effective in modulation of changes caused by noxious stimulation . Consequently, these results clearly indicate that IL- $1 \beta$ plays a role in trigeminal activation. However the results of our Western blot measurement showed that there were not changes in the expression of IL-1及 at the level of TNC four hours after s.c. formalin administration and therefore it is not possible that PROB exerts such effect on this level, but one can not exclude the possibility that PROB affects IL-1 in the periphery, at the inflammation site or in the trigeminal ganglion.

It is important to note that some data suggest that PROB is able to activate some of the transient receptor potential channels, including TRPV2 and TRPA1 which play an important role in pain perception and are present in the trigeminal system . However, the agonist-mediated desensitization of these receptors is also known, which can cause an anti-nociceptive effect . PROB can desensitize the TRPA1 and may act similarly on 
TRPV2 as well, which might account of its anti-nociceptive properties in the present experiments.

It is a well-known fact that PROB can increase the concentration of kynurenic acid an endogenous tryptophan metabolite - in the central nervous system . This phenomenon can be caused by inhibition of organic anion transporters by PROB , which are involved in the transport of kynurenic acid taking place from brain through the blood-brain barrier or by increasing of concentration of tryptophan in the central nervous system . Experimental data suggest that kynurenic acid can affect nociception and elevation in its concentration in the brain can inhibit the trigeminal activation , probably due to an antagonistic effect on ionotropic glutamate and $\alpha 7$-nicotinic acetylcholine receptors or due an agonistic action on G-protein-coupled receptor-35 , which receptors play important role in pain processing and trigeminal activation .

Several lines of evidence confirm the role of oxidative stress in the induction of tissue damage e.g. neuronal activation caused by inflammation in orofacial formalin test . However, in the present study, we could not support this hypothesis, since the NQO1 expression, a marker of oxidative stress, remained unchanged four hours after s.c. formalin injection.- 


\section{Conclusions}

The results of our study provide evidence that pre-treatment with PROB can have anti-nociceptive effects and can modulate the changes induced by trigeminal inflammation in both behavioural and morphological markers in the rat orofacial formalin test. PROB may exert this effect through inhibition of the inflammatory processes which could be through blocking the MRP4, which induces the release of key inflammatory mediators such as PGE2 or through desensitization of TRPA1 and TRPV2 or by enhancing concentration of kynurenic acid in the brain. These properties and the relatively good side effect profile can make $\mathrm{PROB}$ a possible pharmacological candidate in the treatment of trigeminal activation related pain. 


\section{Acknowledgements}

This work was supported by the OTKA KI05077, TÁMOP-4.2.2.A-11/1/KONV-20120052 and the European Union and the State of Hungary, co-financed by the European Social Fund in the framework of TÁMOP 4.2.4.A/2-11-1-2012-0001 "National Excellence Program”, by Hungarian Brain Research Program - Grant No. KTIA_13_NAP-A-III/9 and by the EUROHEADPAIN (FP7-Health 2013-Innovation; Grant no. 602633).

Árpád Párdutz was supported by the Bolyai Scholarship Programme of the Hungarian Academy of Sciences.

The specific contributions of the authors to this research were as follows: Annamária FejesSzabó participated in the design and implementation of experiments, collected data for statistical analysis, interpreted the data and wrote the manuscript, Zsuzsanna Bohár, Enikő Vámos and János Tajti participated in the design of the experiments and in the critical revision of the manuscript, Gábor Nagy-Grócz, Lilla Tar and Borbála Pődör participated in the implementation of the experiments, József Toldi participated in the critical revision of the manuscript, László Vécsei participated in the design of the experiments, in the critical revision of the manuscript and in the making final approval of the version to be published, Árpád Párdutz participated in the conception and design of the experiments, the interpretation of the data and the writing and critical revision of the manuscript.

We thank Mrs. Valéria Vékony for the histotechnical assistance, Anna László, M.Sc. for the help in the statistical analysis and David Durham from England for the linguistic correction of the manuscript. 


\section{List of abbreviations}

CGRP: calcitonin gene-related peptide

IL-1 $\beta$ : interleukin-1beta

IR: immunoreactive

MRP4: multidrug resistance-associated protein 4

nNOS: neuronal nitric oxide synthase

NQO1: $\quad$ NAD(P)H:quinone oxidoreductase 1

PBS: $\quad$ phosphate-buffered saline

PBST: phosphate-buffered saline containing 1\% Triton X-100

PGE2: prostaglandin E2

PROB: probenecid

s.c.: $\quad$ subcutaneous

TNC: $\quad$ caudal part of spinal trigeminal nucleus

TRPV1: transient receptor potential channel subtype V member 1

TRPV2: transient receptor potential channel subtype V member 2

TRPA1: transient receptor potential channel subtype A member 1 


\section{Figure legends}

Fig. 1. Diagram showing the time (number of seconds) spent rubbing the injected area with the ipsilateral fore- or hindpaw in each block of $3 \mathrm{~min}$ in all 4 subgroups. In the PlaceboForm subgroup (black diamonds), the two phases of formalin action are clearly distinguishable. The rubbing activity in the first $(* * * p<0.001)$ and in the fifth to eleventh $(* * \mathrm{p}<0.01 ; * * * \mathrm{p}<0.001)$ blocks was significantly higher in the Placebo-Form subgroup (black diamonds) than in the Placebo-Phys subgroup (dark-grey squares). The PROB pre-treatment significantly reduced the formalin-induced nociceptive behaviour in each of the above-mentioned blocks $\left({ }^{\#} \mathrm{p}<0.01\right.$; $\left.{ }^{\# \#} \mathrm{p}<0.001\right)$ in the PROB-Form subgroup (black triangles) relative to the Placebo-Form subgroup. There was no significant difference between the findings on the Placebo-Phys and PROB-Phys animals in any block.

Fig. 2. Diagrams showing the rubbing activity in the first (A) and the second (B) phase in all 4 subgroups. In the Placebo group, the subcutaneous formalin injection (Placebo-Form) induced a significant increase in rubbing activity in both the first and the second phase $(* * * p<0.001)$ as compared with that in the saline-treated animals (Placebo-Phys). In both phases, pre-treatment with PROB had a significant effect on mitigating the formalininduced increase in the time spent in rubbing $(* * p<0.01 ; * * * p<0.001)$ as compared with the Placebo-Form subgroup. 
Fig. 3. (A) c-Fos-immunoreactive (IR) neurones (black arrow) in the superficial laminae of a transverse section of medulla containing the caudal part of the spinal trigeminal nucleus (TNC) in the Placebo-Form subgroup. Subcutaneous formalin injection resulted in a higher number of c-Fos-IR neurones on the ipsilateral side than on the contralateral side. Scale bar: $500 \mu \mathrm{m}$. (B) c-Fos-IR neurones on the formalin-treated ipsilateral sides of sections in the TNC in the two subgroups. The formalin-induced increase in IR cells was more prominent on the ipsilateral side in the Placebo-Form subgroup (B/a) than in the probenecid (PROB) (B/b)-pretreated animals. Scale bar: $200 \mu \mathrm{m}$. (C) Diagram showing the mean number of c-Fos-IR cells in the superficial laminae of the TNC at different levels along the rostrocaudal axis (mean \pm S.E.M, $\mathrm{n}=\mathbf{9}$ per subgroup). In the PlaceboForm subgroup, formalin produced a significantly higher number of c-Fos-IR neurones on the formalin-treated side of the $\mathrm{TNC}$ as compared with the contralateral side at different levels along the rostrocaudal axis (between -0.3 and $-3.3 \mathrm{~mm}$ ), in accordance with the somatotopic representation $(* * \mathrm{p}<0.01 ; * * * \mathrm{p}<0.001)$. PROB pre-treatment significantly decreased the effect of formalin between -0.3 and $-2.4 \mathrm{~mm}\left({ }^{\#} \mathrm{p}<0.05\right.$; $\left.{ }^{\# \#} \mathrm{p}<0.01 ;{ }^{\# \#} \mathrm{p}<0.001\right)$.

Fig. 4. (A) Immunostained transverse sections of medulla containing the caudal part of the spinal trigeminal nucleus (TNC) from the Placebo-Form and probenecid (PROB)-Form subgroup. Black arrow shows the neuronal nitric-oxide synthase (nNOS)-immunoreactive (IR) neurones in the superficial area of the TNC. On the ipsilateral side (A/b), more nNOS-IR neurones can be observed than on the contralateral side (A/a) in the Placebo-Form subgroup. After formalin injection, there is a lower number of nNOS-IR neurones on the ipsilateral side in the probenecid (PROB)-Form subgroup (A/d) than in the Placebo-Form subgroup 
$(A / b)$. In these two subgroups, difference between the contralateral sides cannot be observed. Scale bar: $200 \mu \mathrm{m}$. (B) Diagram showing the mean number of nNOS-IR cells in the superficial area of the TNC at different levels along the rostrocaudal axis (mean \pm S.E.M, $n=5$ per subgroup). Formalin produced a significant increase in number of nNOS-IR neurones on the formalin-treated side of the TNC in the Placebo-Form subgroup as compared with the contralateral side at different levels along the rostrocaudal axis (between -2.1 and -2.7 and between -3.3 and $-3.9 \mathrm{~mm}$; $\left.{ }^{*} \mathbf{p}<0.05 ; * * p<0.01\right)$. The effect of formalin was significantly decreased by pretreatment with PROB between -2.1 and -2.7 and between -3.3 and $-3.9 \mathrm{~mm}$ $\left({ }^{\#} \mathbf{p}<0.05 ;{ }^{\# \#} p<0.01\right)$. There was no significant difference between the contralateral sides. 


\section{References}

[1] Burnell JM, Kirby WM. Effectiveness of a new compound, benemid, in elevating serum penicillin concentrations. J Clin Invest 1951; 30(7): 697-700.

[2] Gutman AB, Yu TF. Benemid (p-di-n-propylsulfamyl)-benzoic acid) as uricosuric agent in chronic gouty arthritis. Trans Assoc Am Physicians 1951; 64: 279-88.

[3] van Aubel RA, Smeets PH, Peters JG, Bindels RJ, Russel FG. The MRP4/ABCC4 gene encodes a novel apical organic anion transporter in human kidney proximal tubules: putative efflux pump for urinary cAMP and cGMP. J Am Soc Nephrol 2002; 13(3): 595-603.

[4] Reid G, Wielinga P, Zelcer N, van der Heijden I, Kuil A, de Haas M, Wijnholds J, Borst $\mathrm{P}$. The human multidrug resistance protein MRP4 functions as a prostaglandin efflux transporter and is inhibited by nonsteroidal antiinflammatory drugs. Proc Natl Acad Sci U S A 2003; 100(16): 9244-9.

[5] Silverman W, Locovei S, Dahl G. Probenecid, a gout remedy, inhibits pannexin 1 channels. Am J Physiol Cell Physiol 2008; 295(3): C761-7.

[6] Silverman WR, de Rivero Vaccari JP, Locovei S, Qiu F, Carlsson SK, Scemes E, Keane RW, Dahl G. The pannexin 1 channel activates the inflammasome in neurons and astrocytes. J Biol Chem 2009; 284(27): 18143-51.

[7] McClenaghan C, Zeng F, Verkuyl JM. TRPA1 agonist activity of probenecid desensitizes channel responses: consequences for screening. Assay Drug Dev Technol 2012; 10(6): 533-41.

[8] Bang S, Kim KY, Yoo S, Lee SH, Hwang SW. Transient receptor potential V2 expressed in sensory neurons is activated by probenecid. Neurosci Lett 2007; 425(2): $120-5$. 
[9] Shimosato G, Amaya F, Ueda M, Tanaka Y, Decosterd I, Tanaka M. Peripheral inflammation induces up-regulation of TRPV2 expression in rat DRG. Pain 2005; 119(1-3): 225-32.

[10] Bandell M, Story GM, Hwang SW, Viswanath V, Eid SR, Petrus MJ, Earley TJ, Patapoutian A. Noxious cold ion channel TRPA1 is activated by pungent compounds and bradykinin. Neuron 2004; 41(6): 849-57.

[11] Mercado J, Gordon-Shaag A, Zagotta WN, Gordon SE. Ca2+-dependent desensitization of TRPV2 channels is mediated by hydrolysis of phosphatidylinositol 4,5-bisphosphate. J Neurosci 2010; 30(40): 13338-47.

[12] Clavelou P, Pajot J, Dallel R, Raboisson P. Application of the formalin test to the study of orofacial pain in the rat. Neurosci Lett 1989; 103(3): 349-53.

[13] D'Andrea G, Leon A. Pathogenesis of migraine: from neurotransmitters to neuromodulators and beyond. Neurol Sci 2010; 31 Suppl 1: S1-7.

[14] Russell MB, Rasmussen BK, Thorvaldsen P, Olesen J. Prevalence and sex-ratio of the subtypes of migraine. Int J Epidemiol 1995; 24(3): 612-8.

[15] Clavelou P, Dallel R, Orliaguet T, Woda A, Raboisson P. The orofacial formalin test in rats: effects of different formalin concentrations. Pain 1995; 62(3): 295-301.

[16] Hunt SP, Pini A, Evan G. Induction of c-fos-like protein in spinal cord neurons following sensory stimulation. Nature 1987; 328(6131): 632-4.

[17] Wang LG, Li HM, Li JS. Formalin induced FOS-like immunoreactive neurons in the trigeminal spinal caudal subnucleus project to contralateral parabrachial nucleus in the rat. Brain Res 1994; 649(1-2): 62-70.

[18] Pardutz A, Krizbai I, Multon S, Vecsei L, Schoenen J. Systemic nitroglycerin increases nNOS levels in rat trigeminal nucleus caudalis. Neuroreport 2000; 11(14): 3071-5. 
[19] Raboisson P, Dallel R. The orofacial formalin test. Neurosci Biobehav Rev 2004; 28(2): 219-26.

[20] Tjolsen A, Berge OG, Hunskaar S, Rosland JH, Hole K. The formalin test: an evaluation of the method. Pain 1992; 51(1): 5-17.

[21] Svensson CI, Yaksh TL. The spinal phospholipase-cyclooxygenase-prostanoid cascade in nociceptive processing. Annu Rev Pharmacol Toxicol 2002; 42: 553-83.

[22] Watkins LR, Martin D, Ulrich P, Tracey KJ, Maier SF. Evidence for the involvement of spinal cord glia in subcutaneous formalin induced hyperalgesia in the rat. Pain 1997; 71(3): 225-35.

[23] Viggiano E, Monda M, Viggiano A, Aurilio C, De Luca B. Persistent facial pain increases superoxide anion production in the spinal trigeminal nucleus. Mol Cell Biochem 2010; 339(1-2): 149-54.

[24] Paxinos G, Watson C. The rat brain in stereotaxic coordinates. 6th ed. Amsterdam: Elsevier 2007.

[25] Ferreira SH, Nakamura M. I - Prostaglandin hyperalgesia, a cAMP/Ca2+ dependent process. Prostaglandins 1979; 18(2): 179-90.

[26] Gyires K, Knoll J. Inflammation and writhing syndrome inducing effect of PGE1, PGE2 and the inhibition of these actions. Pol J Pharmacol Pharm 1975; 27(3): 257-64.

[27] Jenkins DW, Sellers LA, Feniuk W, Humphrey PP. Characterization of bradykinininduced prostaglandin E2 release from cultured rat trigeminal ganglion neurones. Eur J Pharmacol 2003; 469(1-3): 29-36.

[28] Moriyama T, Higashi T, Togashi K, Iida T, Segi E, Sugimoto Y, Tominaga T, Narumiya S, Tominaga M. Sensitization of TRPV1 by EP1 and IP reveals peripheral nociceptive mechanism of prostaglandins. Mol Pain 2005; 1: 3. 
[29] Garry MG, Walton LP, Davis MA. Capsaicin-evoked release of immunoreactive calcitonin gene-related peptide from the spinal cord is mediated by nitric oxide but not by cyclic GMP. Brain Res 2000; 861(2): 208-19.

[30] Zhang RX, Mi ZP, Qiao JT. Changes of spinal substance P, calcitonin gene-related peptide, somatostatin, Met-enkephalin and neurotensin in rats in response to formalininduced pain. Regul Pept 1994; 51(1): 25-32.

[31] Neeb L, Hellen P, Boehnke C, Hoffmann J, Schuh-Hofer S, Dirnagl U, Reuter U. IL1beta stimulates COX-2 dependent PGE(2) synthesis and CGRP release in rat trigeminal ganglia cells. PLoS One 2011; 6(3): e17360.

[32] Jenkins DW, Langmead CJ, Parsons AA, Strijbos PJ. Regulation of calcitonin generelated peptide release from rat trigeminal nucleus caudalis slices in vitro. Neurosci Lett $2004 ; 366(3): 241-4$.

[33] Simmons DL, Botting RM, Hla T. Cyclooxygenase isozymes: the biology of prostaglandin synthesis and inhibition. Pharmacol Rev 2004; 56(3): 387-437.

[34] Gao Y, Duan YZ. Increased COX2 in the trigeminal nucleus caudalis is involved in orofacial pain induced by experimental tooth movement. Anat Rec (Hoboken) 2010; 293(3): 485-91.

[35] Kadoi J, Takeda M, Matsumoto S. Prostaglandin E2 potentiates the excitability of small diameter trigeminal root ganglion neurons projecting onto the superficial layer of the cervical dorsal horn in rats. Exp Brain Res 2007; 176(2): 227-36.

[36] Hoffmann J, Neeb L, Israel H, Dannenberg F, Triebe F, Dirnagl U, Reuter U. Intracisternal injection of inflammatory soup activates the trigeminal nerve system. Cephalalgia 2009; 29(11): 1212-7. 
[37] Myren M, Baun M, Ploug KB, Jansen-Olesen I, Olesen J, Gupta S. Functional and molecular characterization of prostaglandin E2 dilatory receptors in the rat craniovascular system in relevance to migraine. Cephalalgia 2010; 30(9): 1110-22.

[38] Patwardhan AM, Vela J, Farugia J, Vela K, Hargreaves KM. Trigeminal nociceptors express prostaglandin receptors. J Dent Res 2008; 87(3): 262-6.

[39] Price TJ, Patwardhan A, Akopian AN, Hargreaves KM, Flores CM. Modulation of trigeminal sensory neuron activity by the dual cannabinoid-vanilloid agonists anandamide, N-arachidonoyl-dopamine and arachidonyl-2-chloroethylamide. Br J Pharmacol 2004; 141(7): 1118-30.

[40] Jenkins DW, Feniuk W, Humphrey PP. Characterization of the prostanoid receptor types involved in mediating calcitonin gene-related peptide release from cultured rat trigeminal neurones. Br J Pharmacol 2001; 134(6): 1296-302.

[41] Matsumura K, Watanabe Y, Imai-Matsumura K, Connolly M, Koyama Y, Onoe H. Mapping of prostaglandin E2 binding sites in rat brain using quantitative autoradiography. Brain Res 1992; 581(2): 292-8.

[42] Antonova M, Wienecke T, Olesen J, Ashina M. Prostaglandin E(2) induces immediate migraine-like attack in migraine patients without aura. Cephalalgia 2012; 32(11): 82233.

[43] Bruzzone R, Hormuzdi SG, Barbe MT, Herb A, Monyer H. Pannexins, a family of gap junction proteins expressed in brain. Proc Natl Acad Sci U S A 2003; 100(23): 13644-9.

[44] Zoidl G, Petrasch-Parwez E, Ray A, Meier C, Bunse S, Habbes HW, Dahl G, Dermietzel R. Localization of the pannexin1 protein at postsynaptic sites in the cerebral cortex and hippocampus. Neuroscience 2007; 146(1): 9-16.

[45] Pelegrin P, Surprenant A. Pannexin-1 mediates large pore formation and interleukin1beta release by the ATP-gated P2X7 receptor. EMBO J 2006; 25(21): 5071-82. 
[46] Takeda M, Tanimoto T, Kadoi J, Nasu M, Takahashi M, Kitagawa J, Matsumoto S. Enhanced excitability of nociceptive trigeminal ganglion neurons by satellite glial cytokine following peripheral inflammation. Pain 2007; 129(1-2): 155-66.

[47] Guo W, Wang H, Watanabe M, Shimizu K, Zou S, LaGraize SC, Wei F, Dubner R, Ren K. Glial-cytokine-neuronal interactions underlying the mechanisms of persistent pain. J Neurosci 2007; 27(22): 6006-18.

[48] Takahashi K, Watanabe M, Suekawa Y, Ito G, Inubushi T, Hirose N, Murasaki K, Hiyama S, Uchida T, Tanne K. IL-1beta in the trigeminal subnucleus caudalis contributes to extra-territorial allodynia/hyperalgesia following a trigeminal nerve injury. Eur J Pain 2011; 15(5): 467 e1-14.

[49] Oka T, Aou S, Hori T. Intracerebroventricular injection of interleukin-1 beta enhances nociceptive neuronal responses of the trigeminal nucleus caudalis in rats. Brain Res 1994; 656(2): 236-44.

[50] Huang D, Li S, Dhaka A, Story GM, Cao YQ. Expression of the transient receptor potential channels TRPV1, TRPA1 and TRPM8 in mouse trigeminal primary afferent neurons innervating the dura. Mol Pain 2012; 8: 66.

[51] Lee SM, Cho YS, Kim TH, Jin MU, Ahn DK, Noguchi K, Bae YC. An ultrastructural evidence for the expression of transient receptor potential ankyrin 1 (TRPA1) in astrocytes in the rat trigeminal caudal nucleus. J Chem Neuroanat 2012; 45(1-2): 45-9.

[52] Yamamoto Y, Hatakeyama T, Taniguchi K. Immunohistochemical colocalization of TREK-1, TREK-2 and TRAAK with TRP channels in the trigeminal ganglion cells. Neurosci Lett 2009; 454(2): 129-33.

[53] Akopian AN, Ruparel NB, Jeske NA, Patwardhan A, Hargreaves KM. Role of ionotropic cannabinoid receptors in peripheral antinociception and antihyperalgesia. Trends Pharmacol Sci 2009; 30(2): 79-84. 
[54] Materazzi S, Benemei S, Fusi C, Gualdani R, De Siena G, Vastani N, Andersson DA, Trevisan G, Moncelli MR, Wei X, Dussor G, Pollastro F, Patacchini R, Appendino G, Geppetti P, Nassini R. Parthenolide inhibits nociception and neurogenic vasodilatation in the trigeminovascular system by targeting the TRPA1 channel. Pain 2013; 154(12): $2750-8$.

[55] Leklem JE. Quantitative aspects of tryptophan metabolism in humans and other species: a review. Am J Clin Nutr 1971; 24(6): 659-72.

[56] Vecsei L, Miller J, MacGarvey U, Beal MF. Kynurenine and probenecid inhibit pentylenetetrazol- and NMDLA-induced seizures and increase kynurenic acid concentrations in the brain. Brain Res Bull 1992; 28(2): 233-8.

[57] Perwitasari O, Yan X, Johnson S, White C, Brooks P, Tompkins SM, Tripp RA. Targeting organic anion transporter 3 with probenecid as a novel anti-influenza a virus strategy. Antimicrob Agents Chemother 2013; 57(1): 475-83.

[58] Chiba S, Ikawa T, Takeshita H, Ichiba K, Sagi M, Mukai T, Anzai N. Interactions of human organic anion transporter 1 (hOAT1) with substances associated with forensic toxicology. Leg Med (Tokyo) 2011; 13(4): 180-5.

[59] Colin-Gonzalez AL, Santamaria A. Probenecid: an emerging tool for neuroprotection. CNS Neurol Disord Drug Targets 2013; 12(7): 1050-65.

[60] van Wijk M, Sebens JB, Korf J. Probenecid-induced increase of 5-hydroxytryptamine synthesis in rat brain, as measured by formation of 5-hydroxytryptophan. Psychopharmacology (Berl) 1979; 60(3): 229-35.

[61] Nasstrom J, Karlsson U, Post C. Antinociceptive actions of different classes of excitatory amino acid receptor antagonists in mice. Eur J Pharmacol 1992; 212(1): 219. 
[62] Mecs L, Tuboly G, Nagy E, Benedek G, Horvath G. The peripheral antinociceptive effects of endomorphin-1 and kynurenic acid in the rat inflamed joint model. Anesth Analg 2009; 109(4): 1297-304.

[63] Pardutz A, Fejes A, Bohar Z, Tar L, Toldi J, Vecsei L. Kynurenines and headache. J Neural Transm 2012; 119(2): 285-96.

[64] Vecsei L, Szalardy L, Fulop F, Toldi J. Kynurenines in the CNS: recent advances and new questions. Nat Rev Drug Discov 2013; 12(1): 64-82.

[65] Vamos E, Pardutz A, Varga H, Bohar Z, Tajti J, Fulop F, Toldi J, Vecsei L. 1kynurenine combined with probenecid and the novel synthetic kynurenic acid derivative attenuate nitroglycerin-induced nNOS in the rat caudal trigeminal nucleus. Neuropharmacology 2009; 57(4): 425-9.

[66] Vamos E, Fejes A, Koch J, Tajti J, Fulop F, Toldi J, Pardutz A, Vecsei L. Kynurenate derivative attenuates the nitroglycerin-induced CamKIIalpha and CGRP expression changes. Headache 2010; 50(5): 834-43.

[67] Birch PJ, Grossman CJ, Hayes AG. Kynurenate and FG9041 have both competitive and non-competitive antagonist actions at excitatory amino acid receptors. Eur J Pharmacol $1988 ; 151(2): 313-5$.

[68] Kessler M, Terramani T, Lynch G, Baudry M. A glycine site associated with N-methylD-aspartic acid receptors: characterization and identification of a new class of antagonists. J Neurochem 1989; 52(4): 1319-28.

[69] Pereira EF, Hilmas C, Santos MD, Alkondon M, Maelicke A, Albuquerque EX. Unconventional ligands and modulators of nicotinic receptors. J Neurobiol 2002; 53(4): 479-500. 
[70] Wang J, Simonavicius N, Wu X, Swaminath G, Reagan J, Tian H, Ling L. Kynurenic acid as a ligand for orphan G protein-coupled receptor GPR35. J Biol Chem 2006; 281(31): 22021-8.

[71] Fejes A, Pardutz A, Toldi J, Vecsei L. Kynurenine metabolites and migraine: experimental studies and therapeutic perspectives. Curr Neuropharmacol 2011; 9(2): $376-87$.

[72] Yeo JF, Ling SF, Tang N, Ong WY. Antinociceptive effect of CNS peroxynitrite scavenger in a mouse model of orofacial pain. Exp Brain Res 2008; 184(3): 435-8.

[73] Ali S, Diwakar G, Pawa S. Paraquat induces different pulmonary biochemical responses in Wistar rats and Swiss mice. Chem Biol Interact 2000; 125(2): 79-91.

[74] Tomita M, Okuyama T, Katsuyama H, Hidaka K, Otsuki T, Ishikawa T. Gene expression in rat lungs during early response to paraquat-induced oxidative stress. Int $\mathbf{J}$ Mol Med 2006; 17(1): 37-44. 